\title{
ON THE HYPERBOLIC CAPACITY AND CONFORMAL MAPPING ${ }^{1}$
}

\author{
CH. POMMERENKE
}

1. Let $E$ be a compact set in $D=\{|z|<1\}$. Tsuji [6] has introduced the hyperbolic capacity of $E$ which can be defined by

$$
\operatorname{caph} E=\lim _{n \rightarrow \infty} \max _{z_{0}, \ldots, z_{n} \in E} \prod_{\mu=0}^{n} \prod_{p \neq \mu}\left|\frac{z_{\mu}-z_{\nu}}{1-\bar{z}_{\nu} z_{\mu}}\right|^{1 / n(n+1)} .
$$

Also,

$$
\min _{\mathcal{f}} \max _{z \in \mathbb{B}}|f(z)|^{1 / n} \rightarrow \operatorname{caph} E
$$

as $n \rightarrow \infty$ where the minimum is taken over all functions

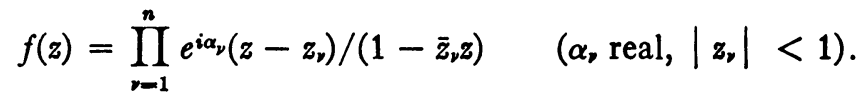

We shall first obtain another formula for caph $E$. Leja [1] has proved an analogous formula for the capacity of a plane set.

Lemma. Let $E$ be a compact set in $D$. For each $n=1,2, \cdots$ choose $n+1$ points $z_{0}, \cdots, z_{n}$ in $E$ such that

$$
\prod_{\mu=0}^{n} \prod_{\nu \neq \mu}\left|z_{\mu}-z_{\nu}\right| /\left|1-\bar{z}_{\nu} z_{\mu}\right|
$$

becomes maximal. Numerate these points so that

$$
\begin{aligned}
A_{n} & =\prod_{\nu=1}^{n}\left|z_{0}-z_{\nu}\right| /\left|1-\bar{z}_{\nu} z_{0}\right| \\
& =\min _{\mu} \prod_{\nu \neq \mu}\left|z_{\mu}-z_{\nu}\right| /\left|1-\bar{z}_{\nu} z_{\mu}\right| .
\end{aligned}
$$

If

$$
f_{n}(z)=\prod_{\nu=1}^{n} \frac{1-\bar{z}_{\nu}}{1-z_{\nu}} \frac{z-z_{\nu}}{1-\bar{z}_{\nu} z}
$$

then

Received by the editors August 1, 1962.

1 Research supported by the National Science Foundation Grant No. G-18837. 


$$
\max _{z \in E}\left|f_{n}(z)\right|=A_{n}
$$

and, as $n \rightarrow \infty$

$$
A_{n}^{1 / n} \rightarrow \operatorname{caph} E .
$$

Proof. For $\left|\zeta_{1}\right|<1,\left|\zeta_{2}\right|<1$ we write $\left[\zeta_{1}, \zeta_{2}\right]=\left|\zeta_{1}-\zeta_{2}\right| /\left|1-\bar{\zeta}_{2} \zeta_{1}\right|$. Let $z \in E$. Comparing the system $z, z_{1}, \cdots, z_{n}$ of points in $E$ with the maximal system $z_{0}, z_{1}, \cdots, z_{n}$ we see that

$$
\begin{aligned}
& 1 \cdot\left[z, z_{1}\right] \quad \cdots\left[z, z_{n}\right] \quad 1 \cdot\left[z_{0}, z_{1}\right] \quad \cdots\left[z_{0}, z_{n}\right] \\
& {\left[z_{1}, z\right] \cdot 1 \cdots\left[z_{1}, z_{n}\right] \leqq\left[z_{1}, z_{0}\right] \cdot 1 \cdots\left[z_{1}, z_{n}\right]} \\
& {\left[z_{n}, z\right]\left[z_{n}, z_{1}\right] \cdots \dot{1} \quad\left[z_{n}, z_{0}\right]\left[z_{n}, z_{1}\right] \cdots \dot{1}}
\end{aligned}
$$

Hence $\left|f_{n}(z)\right| \leqq A_{n}$, with equality for $z=z_{0}$, which proves (6). Since $f_{n}$ has the form (3) it follows that $\min _{f} \max _{z \in E}|f(z)| \leqq A_{n}$. Therefore by (2)

$$
\liminf _{n \rightarrow \infty} A_{n}^{1 / n} \geqq \operatorname{caph} E .
$$

On the other hand, (4) implies

$$
A_{n}^{n+1} \leqq \prod_{\mu=0}^{n} \prod_{\nu \neq \mu}\left[z_{\mu}, z_{\nu}\right] .
$$

Hence (1) shows that $\lim \sup _{n \rightarrow \infty} A_{n}^{1 / n} \leqq \operatorname{caph} E$, and the Lemma follows.

2. Let $E$ be a compact set in $D=\{|z|<1\}$. Then $D \backslash E$ is an open set of which exactly one component region $G$ has $\{|z|=1\}$ as part of the boundary. I shall give an elementary proof of the following theorem.

Theorem 1. Let $\rho=$ caph $E>0$. If $f_{n}(z)$ is defined by (5) then

$$
g(z)=\lim _{n \rightarrow \infty} f_{n}(z)^{1 / n}
$$

exists locally uniformly in $H=G \cup\{1 \leqq|z|<r\}$ for some $r>1$, and $g(z)$ is the smallest function satisfying

(a) $g(z)$ is locally analytic ${ }^{2}$ and of single-valued modulus in $H$,

(b) $|g(z)|=1$ for $|z|=1$,

(c) $1 \geqq|g(z)| \geqq \rho$ for $z \in G$,

that is, if $h(z)$ also satisfies these three conditions then $|g(z)| \leqq|h(z)|$ for $z \in G$.

2 This means that $g(z)$ is analytic on the universal covering surface of $H$. 
Furthermore, $g(1)=1$ and

$$
\int_{0}^{2 \pi} d \arg g\left(e^{i \theta}\right)=2 \pi
$$

If $\zeta$ is a boundary point of $G$ that lies on a continuum contained in $E$ then $|g(z)| \rightarrow \rho$ for $z \rightarrow \zeta, z \in G$.

Finally, if $E$ is a continuum then $\rho>0$, and $w=g(z)$ maps $G$ conformally and one-to-one onto $\{\rho<|w|<1\}$.

REMARKS. Let

$$
\omega(z)=\log \left(\rho^{-1}|g(z)|\right) / \log \rho^{-1} .
$$

Then Theorem 1 shows that $\omega(z)$ is the smallest function satisfying

$\left(a^{\prime}\right) \omega(z)$ is single-valued and harmonic in $H$,

(b') $\omega(z)=1$ for $|z|=1$,

(c') $1 \geqq \omega(z) \geqq 0$ for $z \in G$,

that is, if $\nu(z)$ also satisfies these three conditions then $\omega(z) \leqq \nu(z)$. If the boundary of $G$ consists of a finite number of nondegenerate continua then $\omega(z)=0$ on the boundary points of $G$ that lie in $D$. Hence $\omega(z)$ is the harmonic measure of $\{|z|=1\}$ with respect to $G$. By (8),

$$
1 / \log \rho^{-1}=\left.\frac{1}{2 \pi} \int_{0}^{2 \pi} \frac{\partial}{\partial r} \omega\left(r e^{i \theta}\right)\right|_{r=1} d \theta .
$$

Of course, we could have started with the harmonic measure and then proved (9). But the method applied here is simpler and more constructive. It does not use set-functions, the solvability of the Dirichlet problem or the Riemann mapping theorem. The existence of a function that maps a doubly-connected region onto an annulus is established (see also [4]).

The following proof uses (with some simplifications) the method of extremal points developed by Leja $[1 ; 2 ; 3]$.

Proof. a. The Lagrange interpolation formula shows that

$$
\sum_{\mu=0}^{n}\left(\prod_{\nu \neq \mu} \frac{z-z_{\nu}}{z_{\mu}-z_{\nu}} \cdot \prod_{\nu=1}^{n}\left(1-\bar{z}_{\nu} z_{\mu}\right)\right)=\prod_{\nu=1}^{n}\left(1-\bar{z}_{\nu} z\right) .
$$

Hence

$$
\max _{\mu}\left(\prod_{\nu \neq \mu}\left|\frac{z-z_{\nu}}{z_{\mu}-z_{\nu}}\right| \cdot \prod_{\nu=1}^{n}\left|\frac{1-\bar{z}_{\nu} z_{\mu}}{1-\bar{z}_{\nu} z}\right|\right) \geqq \frac{1}{n+1} \cdot
$$

Let $q(z)=\min _{\zeta_{1}, \zeta_{2} \in E}\left|z-\zeta_{1}\right| /\left|z-\zeta_{2}\right|($ for $z \in G)$. Since $E \subset\{|z| \leqq a\}$ for some $a<1$ it follows that 


$$
\max _{\mu}\left(\prod_{\nu=1}^{n}\left|\frac{z-z_{\nu}}{1-\bar{z}_{\nu} z}\right| \cdot \prod_{\nu \neq \mu}\left|\frac{1-\bar{z}_{\nu} z_{\mu}}{z_{\mu}-z_{\nu}}\right|\right) \geqq \frac{\left(1-a^{2}\right) q(z)}{2(n+1)},
$$

and because of (4)

$$
\left|f_{n}(z)\right| \geqq \frac{\left(1-a^{2}\right) q(z)}{2(n+1)} A_{n} .
$$

We put $r=2 /(1+a)>1$. Since $\left|z-z_{\nu}\right| /\left|1-\bar{z}_{\nu} z\right| \leqq(r+a) /(1-a r)$ $<4 /(1-a)$ for $|z| \leqq r$, (5) shows that

$$
\left|f_{n}(z)\right|^{1 / n}<4 /(1-a) \quad(|z| \leqq r) .
$$

b. Let $H=G \cup\{1 \leqq|z| \leqq r\}$ and $g_{n}(z)=f_{n}(z)^{1 / n}$. The functions $g_{n}(z)$ are locally analytic in $H$, and $\left|g_{n}(z)\right|$ is single-valued. By (11) and Montel's theorem we can find a sequence $n_{k}$ such that $g_{n_{k}}(z)$ converges locally uniformly in $H$. Let $g(z)$ be the limit function. Since by the Lemma $A_{n}^{1 / n} \rightarrow \rho$, inequality (10) implies $|g(z)| \geqq \rho$. Also $|g(z)|=1$ for $|z|=1$ so that $g(z)$ satisfies (a), (b) and (c).

Let $h(z)$ be any function satisfying these three conditions, and let $z^{*}$ be a point in $G$. Given $\epsilon>0$ we choose a fixed $k$ so large that $\left|g_{n_{k}}\left(z^{*}\right)\right|>e^{-\epsilon}\left|g\left(z^{*}\right)\right|$. Since $\rho>0$ we can take $k$ so that also $A_{n_{k}}^{1 / n_{k}}<\rho e^{e}$. Then it follows from (6) that $\left|g_{n_{k}}(z)\right| \leqq \rho e^{e}$ for $z \in E$. We choose analytic curves in $G$ so near to $E$ that their union $C$ separates $E$ from $z^{*}$ and from $\{|z|=1\}$, and that $\left|g_{n_{k}}(z)\right| \leqq \rho e^{2 \epsilon}$ for $z \in C$. Because $|h(z)|$ $\geqq \rho$ for $z \in G$,

$$
\left|g_{n_{k}}(z)\right| /|h(z)| \leqq \rho e^{2 e} / \rho=e^{2 e}
$$

for $z \in C$. Since the left side is $=1$ for $|z|=1$ it follows from the maximum principle that the inequality holds also for $z=z^{*}$. Hence

$$
\left|g\left(z^{*}\right)\right|<e^{\epsilon}\left|g_{n_{k}}\left(z^{*}\right)\right| \leqq e^{3 \epsilon}\left|h\left(z^{*}\right)\right|
$$

for every $\epsilon>0$ and therefore $\left|g\left(z^{*}\right)\right| \leqq\left|h\left(z^{*}\right)\right|$.

Since $f_{n}(1)=1$ we obtain $g(1)=1$, and by the argument principle

$$
\int_{0}^{2 \pi} d \arg g_{n}\left(e^{i \theta}\right)=\frac{1}{n} \int_{0}^{2 \pi} d \arg f_{n}\left(e^{i \theta}\right)=2 \pi
$$

from which (8) follows.

If $g_{n}(z)$ did not converge there would be a limit function $h(z) \not \equiv g(z)$ for some other subsequence of $g_{n}$ as Montel's theorem shows. From what we have proved it follows that $|h(z)| \geqq|g(z)|$. Reversing the roles of $g$ and $h$ we also get $|g(z)| \geqq|h(z)|$. Hence $|h(z)|=|g(z)|$, and $g(1)=h(1)=1$ implies $g(z) \equiv h(z)$. Therefore $g_{n}(z) \rightarrow g(z)$ as $n \rightarrow \infty$.

c. We assume now that $E$ is a continuum. We do not know yet that 
$\rho>0$. If $\rho=0$ then $\lim g_{n}(z)$ might not exist. In this case let $g(z)$ be the limit function in $H$ for some convergent subsequence of $g_{n}$ which exists by (11). The region $H$ is doubly connected, and every simply closed curve in $H$ is homotopic either to a point or the unit circle. Therefore the functions $g(z)$ and $g_{n}(z)$ are single-valued because of (8) and (12). Let $c$ be any point with $\rho<|c|<1$. It follows from (12) that $w=g_{n}(z)$ maps $\{|z|=1\}$ one-to-one onto $\{|w|=1\}$. Hence

$$
\frac{1}{2 \pi i} \int_{|z|=1} \frac{g_{n}^{\prime}(z)}{g_{n}(z)-c} d z=\frac{1}{2 \pi i} \int_{|w|=1} \frac{1}{w-c} d w=1 .
$$

We choose analytic curves $C_{m}(m=1,2, \cdots)$ enclosing $E$ so that the regions between $C_{m}$ and $\{|z|=1\}$ approach $G$. By the Lemma we can choose them so that $\left|g_{n}(z)\right|<|c|$ on each $C_{m}$ for sufficiently large $n$. Then

$$
\frac{1}{2 \pi i} \int_{c_{m}} \frac{g_{n}^{\prime}(z)}{g_{n}(z)-c} d z=0 .
$$

Making $n \rightarrow \infty$ we obtain from (13) and (14) that

$$
\frac{1}{2 \pi i} \int_{|z|-1} \frac{g^{\prime}(z)}{g(z)-c} d z-\frac{1}{2 \pi i} \int_{C_{m}} \frac{g^{\prime}(z)}{g(z)-c} d z=1
$$

for all $m$. Hence $g(z)$ assumes the value $c$ exactly once in $G$. Therefore $w=g(z)$ maps $G$ one-to-one onto $\{\rho<|w|<1\}$.

Suppose that $\rho=0$ were true. Then the inverse function $\psi(w)$ of $w=g(z)$ would be analytic and univalent in $\{0<|w| \leqq 1\}$. Since $|\psi(w)|<1$ it would follow that $\psi(w)$ is bounded and univalent in $\{|w| \leqq 1\}$. Since $|\psi(w)|=1$ for $|w|=1$ this would imply that $\psi(w)$ is a linear function and therefore $E$ a point.

d. Let again $E$ be arbitrary and let $\zeta$ be a boundary point lying on a continuum $B$ that is contained in $E$. Let $g_{0}(z)$ be the function that maps the doubly-connected region between $B$ and $\{|z|=1\}$ onto $\left\{\rho_{0}<|w|<1\right\}$. Let $\lambda$ be such that $\rho_{0}^{\lambda}=\rho$. Then $h(z)=g_{0}(z)^{\lambda}$ satisfies the conditions (a), (b) and (c) of Theorem 1. Hence, as we have already proved, $\rho \leqq|g(z)| \leqq|h(z)|=\left|g_{0}(z)\right|^{\lambda}$. Since for simple topological reasons $\left|g_{0}(z)\right| \rightarrow \rho_{0}$ as $z \rightarrow \zeta, z \in G$ it follows that $|g(z)| \rightarrow \rho$.

3. We shall now prove an analogue to a result by Walsh [7] about the ordinary Green's function. We introduce the hyperbolic metric in the unit disk $D$. A circle perpendicular to $\{|z|=1\}$ will be called a geodesic.

TheOREM 2. Let $E$ be a compact set in $D$ with caph $E>0$, and let 
$g(z)$ be the function defined in Theorem 1. Let $L(r)=\{z:|g(z)|=r\}$ (caph $E<r<1)$. Then at every point of $L(r)$ the inner geodesic normal to $L(r)$ intersects the hyperbolically convex hull $K$ of $E$.

REMARKs. The hyperbolically convex hull of $E$ is defined as the smallest closed set $K \supset E$ that is convex in the hyperbolic metric in the sense that together with any two points also the geodesic segment between these two points belongs to $K$. The set $L(r)$ is the union of a finite number of closed analytic curves which may have multiple points though. At the multiple points $g^{\prime}(z)$ vanishes. It is easy to see that all multiple points lie in $K$ (see [8, p. 157]).

Proof. With the notations of Theorem 1 let $L_{n}(r)=\left\{z:\left|f_{n}(z)\right|=r^{n}\right\}$. We first prove that the inner geodesic normal to $L_{n}(r)$ at any $\zeta \in L_{n}(r)$ intersects $K$. Suppose this were false. Then $\zeta \notin K$. By a conformal mapping of the unit disk onto itself we can make $\zeta=0$. Then the inner geodesic normal becomes a straight ray and is separated from $K$ by a line. We may thus assume that $K \subset\{\operatorname{Re} z<0\}$ and that the inner normal lies in $\{\operatorname{Re} z \geqq 0\}$. Writing $z_{\nu}=x_{\nu}+i y_{\nu}$ we have $x_{\nu}<0$. Hence

$$
\begin{aligned}
\left.\frac{d}{d z} \log f_{n}(z)\right|_{z=0} & =\left.\sum_{\nu=1}^{n} \frac{1-\left|z_{\nu}\right|^{2}}{\left(z-z_{\nu}\right)\left(1-\bar{z}_{\nu} z\right)}\right|_{z=0} \\
& =\sum_{\nu=1}^{n} \frac{1-\left|z_{\nu}\right|^{2}}{\left|z_{\nu}\right|^{2}}\left(-x_{\nu}+i y_{\nu}\right) .
\end{aligned}
$$

Therefore $\operatorname{Re} f_{n}^{\prime}(0) / f_{n}(0)>0$, and the inner geodesic normal to $L_{n}(r)=\left\{z: \operatorname{Re} \log f_{n}(z)=n \log r\right\}$ at 0 lies in $\{\operatorname{Re} z<0\}$ (except for the point 0$)$, in contradiction to our assumption. Theorem 2 follows because $f_{n}(z)^{1 / n} \rightarrow g(z)$ locally uniformly in $G$.

4. We shall apply Theorem 2 to obtain a result about the distortion under the conformal mapping of an annulus. It is a generalization of Theorem 6 in [5]. The closure of the region inside $D$ that lies between two geodesics with common endpoint $\zeta$ will be called a geodesic sector of vertex $\zeta$.

THEOREM 3. Let $G$ be a doubly connected region in $D$, with $\{|z|=1\}$ as outer and $E$ as inner boundary. Let $w=g(z)$ be the function that maps $G$ conformally onto $\{\rho<|w|<1\}$ such that $g(1)=1$. Let $S$ be the smallest geodesic sector of vertex 1 that contains $E$, and let $T$ be the component of $S \backslash E$ that contains 1 . If $R$ is the curve that $w=g(z)$ maps onto the interval $(\rho, 1)$ then $R \subset T$. 
By the conformal mapping $z^{*}=(1+z) /(1-z)$ of $D$ onto $\left\{\operatorname{Re} z^{*}>0\right\}$ we see that Theorem 3 is equivalent with

ThEOREM $3^{*}$. Let $G^{*}$ be a doubly connected region in $\left\{\operatorname{Re} z^{*}>0\right\}$ with $\left\{\operatorname{Re} z^{*}=0\right\}$ and $E^{*}$ as boundaries. Let $w=g^{*}(z)$ map $G^{*}$ conformally onto $\{\rho<|w|<1\}$ such that $g^{*}(+\infty)=1$. Let $S^{*}$ be the smallest strip parallel to the real axis that contains $E^{*}$, and let $T^{*}$ be the component of $S^{*} \backslash E^{*}$ that contains $+\infty$. If $R^{*}$ is the curve that $w=g^{*}(z)$ maps onto the interval $(\rho, 1)$ then $R^{*} \subset T^{*}$.

Proof. Theorem 2 shows that all tangents to $R^{*}$ certainly intersect $S^{*}$. If $S^{*}=\left\{a \leqq \operatorname{Im} z^{*} \leqq b\right\}$ it follows that

$$
\lim _{z^{*} \in R^{*}, z^{*} \rightarrow+\infty} \operatorname{Im} z^{*} \leqq b .
$$

Also, all accumulation points of the left end of $R^{*}$ lie on $E^{*}$, hence in $\left\{\operatorname{Im} z^{*} \leqq b\right\}$. Suppose $\max _{z^{*} \in R^{*}} \operatorname{Im} z^{*}>b$. Together with (15) this would imply that the maximum is assumed, say at $z=c$. But $c \notin S^{*}$, and the tangent to $R^{*}$ at $c$ is parallel to $S^{*}$ so that it would not intersect $S^{*}$. Thus we have shown that $\operatorname{Im} z^{*} \leqq b$, and also $\operatorname{Im} z^{*} \geqq a$, for $z^{*} \in S^{*}$. Hence $R^{*} \subset S^{*}$. Since $R^{*}$ is a curve and contains points with large real part, $R^{*}$ has to lie in the component $T^{*}$ of $S^{*} \backslash E^{*}$ that contains the point $+\infty$.

I want to thank the referee for his helpful suggestions.

\section{REFERENCES}

1. F. Leja, Sur les suites des polynomes, les ensembles fermés et la fonction de Green, Ann. Soc. Polon. Math. 12 (1934), 57-71.

2. - Construction de la fonction analytique effectuant la representation conforme d'un domaine plan quelconque sur le cercle, Math. Ann. 111 (1935), 501-504.

3. - Sur une suite de polynômes et la représentation conforme d'un domaine plan quelconque sur les cercle, Ann. Soc. Polon. Math. 14 (1936), 116-134.

4. - Polynômes extrémaux et la représentation conforme des domaines doublement connexes, Ann. Polon. Math. 1 (1954), 13-28.

5. Ch. Pommerenke, Über einige Klassen meromorpher schlichter Funktionen, Math. Z. 78 (1962), 263-284.

6. M. Tsuji, Some metrical theorems on Fuchsian groups, Japan. J. Math. 19 (1947), 484-516.

7. J. L. Walsh, Lemniscates and equipotential curves of Green's function, Amer. Math. Monthly 42 (1935), 1-17.

8. - The location of critical points of analytic and harmonic functions, Amer. Math. Soc., Providence, R. I., 1950.

CORNELl University 